electroclinical sleep discordance, and the frequency of early acquisition of a mature pattern of quiet sleep (continuous, slow-wave sleep) was significantly higher than in the comparison group below conceptional age 45 weeks. The significance of precocious maturity of quiet sleep was unclear, but may indicate accelerated biological development. (Legido $A$ et al. Electroencephalographic and behavioral-state studies in infants of cocaineaddicted mothers. AJDC June 1992; 146:748-752.) (Reprints: Dr. Legido, St. Christopher's Hospital for Children, Section of Neurology, Erie Avenue at Front Street, Philadelphia, PA 19134-1095.)

COMMENT. The authors suggest that the longitudinal assessment of sleep disturbance and its relation to later development might permit tracking of the long-term effects of prenatal exposure to cocaine. Other complications of cocaine exposure in utero are small head circumference, abnormal behavior, cerebral infarction or hemorrhage, seizures and SIDS.

\title{
CEREBELLAR SYMPTOMS IN CRIGLER-NAJJAR TYPE I DISEASE
}

Three children with Crigler-Najjar (CN) type I disease who had cerebellar symptoms as the initial manifestation of kernicterus are reported from the Hopital Antoine Beclere, Clamart Cedex, France. Patient 1, a 3600 gram boy, was admitted with jaundice and a serum total bilirubin of 507 umol/L unconjugated on day 2. After two exchange transfusions and continuous phototherapy the jaundice persisted and a therapeutic trial with phenobarbital was of no benefit. The bilirubin UDPG-T hepatic activity assayed at 3 months was absent. At age 6 years during an episode of fever and headache with respiratory viral infection the jaundice increased and ataxia and dysmetria developed. Liver transplantation was performed with apparent success. In all 3 children the cerebellar symptoms began after an infectious episode or an interruption of phototherapy. The neurotoxicity was associated with an elevated saturation of albumin by bilirubin of $61 \%$ to $75 \%$. In 8 patients without neurotoxicity this parameter remained below $58 \%$. (Labrune $\mathrm{PH}$ et al. Cerebellar symptoms as the presenting manifestations of bilirubin encephalopathy in children with Crigler-Najjar type I disease. Pediatrics April 1992; $199: 768-770$.$) (Reprints: P.H. Labrune, M.D., Service de Pediatrie, Hopital$ antoine Beclere, 157 rue de la Porte de trivaux, 92141 Clamart Cedex, France.)

COMMENT. Cerebellar symptoms are rare features of kernicterus despite the known occurrence of bilirubin staining of the dentate nuclei in pathological studies. The absence of reports of cerebellar involvement in the neonate may be explained by the difficulty in detection of cerebellar signs. Since neurological impairment may be permanent and is potentially preventable, patients with $\mathrm{CN}-1$ disease are candidates for liver transplantation performed when phototherapy is ineffective and prior to the development of kernicterus. Therapy of jaudice in the newborn is reviewed by Newman TB and Maisels MJ in

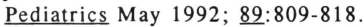

